\title{
Evaluation of p-terphenyl and 2,2" dimethyl-p-terphenyl as wavelength shifters for barium fluoride
}

\author{
T.A. DeVol, D.K. Wehe and G.F. Knoll \\ Department of Nuclear Engineerng, The Unicersity of Michgan, Ann Arbor, MI 48109-2100, USA
}

Received 9 September 1992

p-Terphenyl (PTP) and 2,2" dimethyl-p-terphenyl (DMT) have been investigated for use as wavelength shifters (WLSs) for barıum fluoride $\left(\mathrm{BaF}_{2}\right)$ scintıllation emisssion. These two organic fluors are attractive due to their high quantum efficiency, fast fluorescence decay time, and emissions above the wavelength cut-off of borosilicate glass. Measurements of these WLSs dissolved in cyclohexane are presented. Coincidence time spectra and fluorescence decay spectra measured with $\mathrm{BaF}_{2}$ as the pump source, are shown. The fluorescence decay time and the ratio of fast to slow intensities were determined from the fluorescence decay spectrum. The increase in signal resulting from using a WLS between the borosilicate end-window photomultiplier tube and the $\mathrm{BaF}_{2}$ crystal is measured. The motivation behind this work is the development of a gamma-ray spectrometer sutable for high count rate applications. We are also investigating the possibility of separating the fast and slow emissions of BaF $\mathrm{B}_{2}$ using optical techniques. PTP and DMT both can spectrally separate $\mathrm{BaFe}_{2}$ emissions to a marginal extent.

\section{Introduction}

Barium fluoride $\left(\mathrm{BaF}_{2}\right)$ is moderately radiation hard $[1,2]$ and one of the fastest known inorganic room temperature scintillators [3]. About $20 \%$ of $\mathrm{BaF}_{2}$ emissions $(5 \%$ relative to $\mathrm{Nal}(\mathrm{Tl})$ have a decay time constant of $0.8 \mathrm{~ns}$. A spectrometer utilizing $\mathrm{BaF}_{2}$ typically yields an energy resolution of $11 \%$ full-width-at-halfmaximum (FWHM) at $662 \mathrm{keV}$ [3]. Measuring only the fast emissions of $\mathrm{BaF}_{2}$, the energy resolution is $30 \%$ FWHM at $662 \mathrm{keV}$ [4]. Fig. 1 shows a typical scintillation decay spectrum of $\mathrm{BaF}_{2}$. The very fast $0.8 \mathrm{~ns}$ and the slow 576 ns decay time constants are apparent. Fig. 2 shows a typical emission spectrum of $\mathrm{BaF}_{2}$ when excited with gamma rays. One of the interesting propcrties of $\mathrm{BaF}_{2}$ is that the $220 \mathrm{~nm}$ peak emissions decay with a $0.8 \mathrm{~ns}$ decay time, whereas the $310 \mathrm{~nm}$ peak emissions primarily have a 576 ns decay time. Consequently, separating the emissions in energy (wavelength) results in an approximate separation of the decay times [3].

Borosilicate end-window photomultiplier tubes strongly attenuate light below $300 \mathrm{~nm}$ so use with $\mathrm{BaF}_{2}$ results in detecting only $\approx 38 \%$ of the total emissions, and almost none of the fast cmissions. Fused silica end-window photomultiplier tubes (PMTs) are useful for measuring photons with wavelengths down to $\approx 180$ nm. For some applications the added expense of a fused silica PMT can be avoided by using a wavelength shifters (WLSs) with a borosilicate PMT. This paper demonstrates using WLSs to shift the deep UV $(\lambda<300$ $\mathrm{nm}$ ) emissions of $\mathrm{BaF}_{2}$ while preserving the inherent speed of the scintillator as much as possible. Also, in section 4, detection techniques using p-terphenyl (PTP) or 2, 2" dimethyl-p-terphenyl (DMT) to spectrally separate $\mathrm{BaF}_{2}$ emissions will be discussed.

\section{Background}

Wavelength shifters can be chosen to shift $\mathrm{BaF}_{2}$ emissions above the borosilicate glass wavelength cutoff. Fig. 3 shows the arrangement used for shifting $\mathrm{BaF}_{2}$ emissions, and measuring the changes in the emission and temporal characteristics with the WLS. The WLS absorbs the deep UV emissions from $\mathrm{BaF}_{2}$, $I_{0, \mathrm{BaF}_{2}}(\lambda, t)$, and with probability $Q_{\mathrm{f}}$ (quantum efficiency) reradiates the absorbed energy by emitting photons, $I_{\mathrm{WLS}}\left(\lambda^{\prime}, t\right)$, at a characteristic wavelength and decay time of the organic solute.

Several researchers have used wavelength shifting techniques in various ways to detect the total light output of $\mathrm{BaF}_{2}$ with borosilicate end-window PMTs. The most commonly used method is to vacuum evaporate a fluor onto the face of the scintillator [5-7] that is coupled to the PMT. Vacuum evaporation leaves a whitish translucent film on the scintillator, so only thin films are used in order to minimize the amount of 


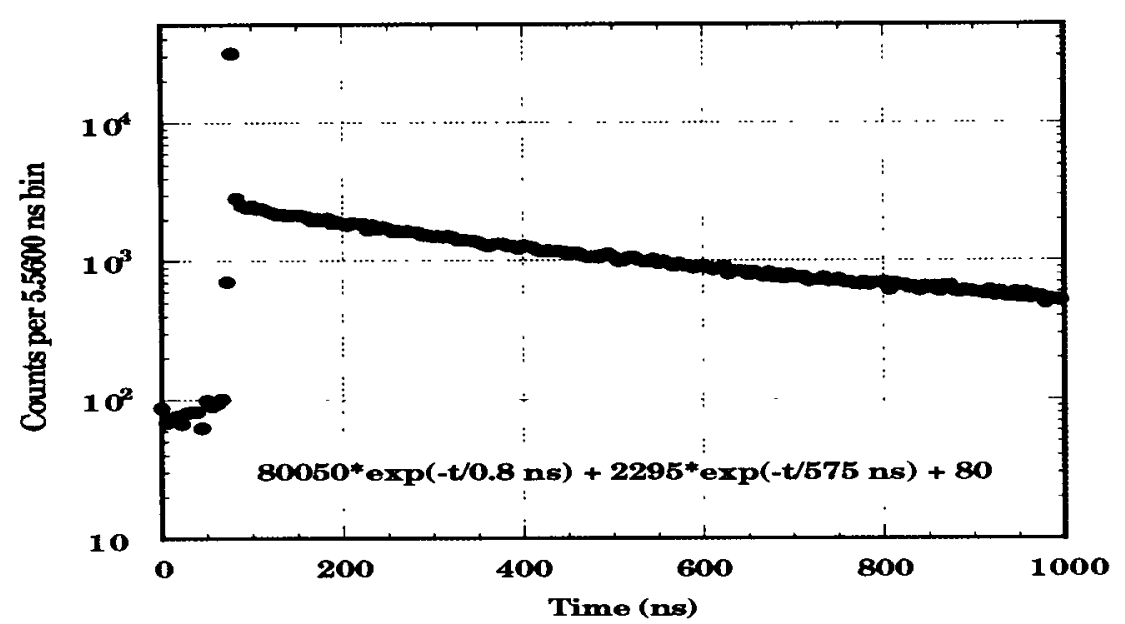

Fig. 1. Scintillation time spectrum of barıum fluoride measured in $0.556 \mathrm{~ns}$ bıns and rebinned to $5.56 \mathrm{~ns} / \mathrm{bin}$ for display.

optical traps and reflection of shifted light. Another technique involves fluors dissolved in a polymer matrix [8]. This technique allows higher concentrations of the fluor to be utilized, is physically more rigid than vacuum evaporation, and forms a medium that is transparent to its own emissions. In this work, liquid WLS solutions were contained in a fused silica cuvette. Although the photon detection geometry is not ideal, the arrangement allows for efficient comparisons of WLSs and results in higher photon yield than vacuum evaporation because the medium is transparent to the solute emissions.

PTP and DMT are two members of the terphenyl group of the p-oligophenylene series. This series is known to have properties appropriate for wavelength shifting $[9,10]$. Fig. 4 shows the optical absorption and fluorescence characteristics of these molecules, demonstrating that they are good candidates for shifting $\mathrm{BaF}_{2}$ emissions above the cut-off wavelength $(\approx 300 \mathrm{~nm})$ of a borosilicate end-window PMT. PTP and DMT have large Stokes shifts, so there is only a slight overlap between the emission and absorption bands (see fig. 4) resulting only small losses from self-absorption.

For high count rate applications, it is advantageous to collect the fast photons and eliminate the slow photons. Several researchers have shown reasonable separation of the $\mathrm{BaF}_{2}$ emissions using a solar blind photocathode PMT [4], doping $\mathrm{BaF}_{2}$ with $\mathrm{La}^{3+}$ [11],

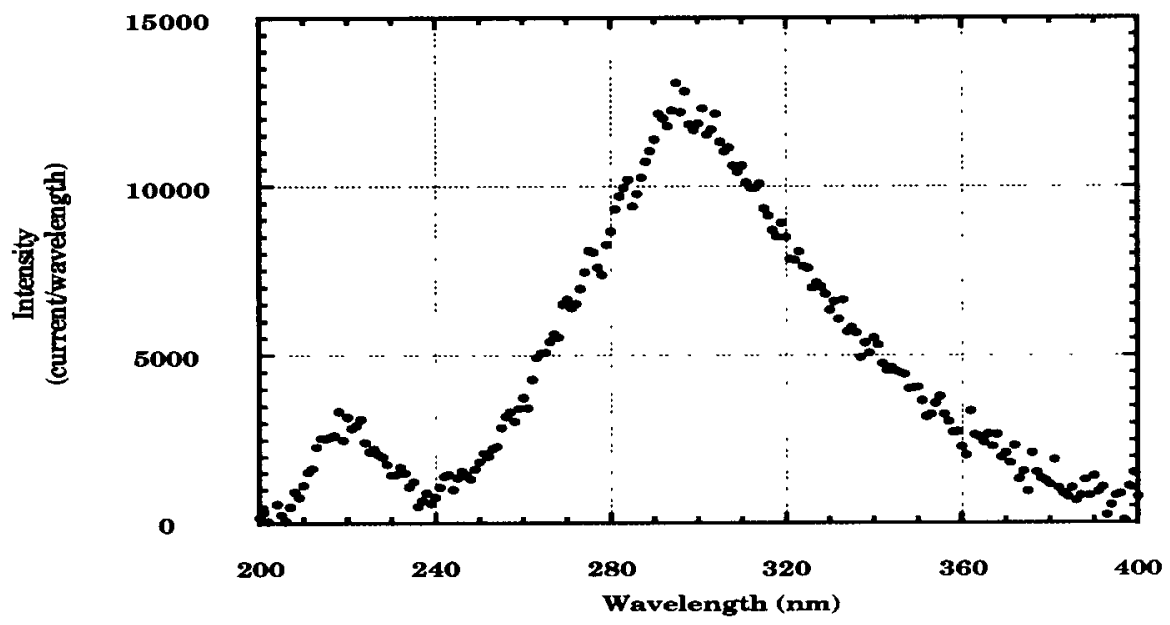

Fig. 2. Barium fluoride emissıon spectrum when excited with $662 \mathrm{keV}$ gamma rays. 


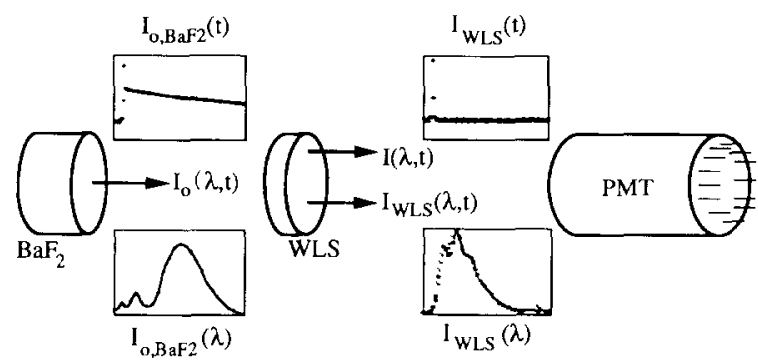

Fig. 3. Schematıc dıagram of a typıcal wavelength shifter.

increasing crystal tempcrature [12] and using fast gated integration [3]. In section 4 we will discuss using PTP and DMT to spectrally separate the $\mathrm{BaF}_{2}$ emissions.

\section{Data acquisition and analysis}

Several propertics are needed to evaluate the effectiveness of an organic fluor for wavelength shifting $\mathrm{BaF}$, emissions. These properties include the molar extinction coefficient, fluorescence emission wavelength, quantum efficiency, and fluorescence decay timc. In this section, we report the results of the measurements of these key parameters for PTP and DMT in cyclohexane.

\subsection{Absorption measurements}

Absorption data for organic fluors are generally unavailable below $\approx 250 \mathrm{~nm}$ [13]. However, measure- ments of the absorption characteristics in the shorter wavelength region are necessary to properly evaluate the WLS to be used with $\mathrm{BaF}_{2}$. Typical spectrophotometers cannot measure the absorption of WLS solutes because the instrument has no means of discriminating between transmitted beam and samplc fluorescence. For this reason, the method illustrated in fig. 5 was used. An Oriel Spectra-Pro scanning monochromator equipped with a $250 \mathrm{~nm}$ blaze, 1200 grooves $/ \mathrm{mm}$ grating, with entrance and cxit slits set to have a resolution better than $0.1 \mathrm{~nm}$, was used for these measurements. The WLS sample was placed in a lowfluorescence fused silica cuvctte and illuminated with the broad band emissions of a calibrated deuterium lamp. The transmitted lamp intensity, $I_{0}(\lambda)$, was recorded in $1 \mathrm{~nm}$ steps with the cuvette filled only with cyclohexane. Next a measurement of the transmitted light intensity through the WLS solute dissolved in cyclohexane, $I(\lambda)$, was recorded under the same conditions. The ratio. $I_{0}(\lambda) / I(\lambda)$, mcasured at each incremental wavelength was used to determine the molar extınction coefficient, $\epsilon(\lambda)$, using the Beer-Lambert law.

$I(\lambda)=I_{0}(\lambda) 10^{-\epsilon b r}$.

where $b$ is the concentration of solute in M (mole/l), $x$ is the light path length in $\mathrm{cm}$, and $\epsilon(\lambda)$ has units of $1 /$ mole $\mathrm{cm}$. The measured values of $\epsilon(\lambda)$ are shown using hollow data points in fig. 4 . Very dilute solutions $\left(\approx 4.5 \times 10^{-5} \mathrm{M}\right)$ were used to decrease the contributions from concentration quenching and self-absorption [14].

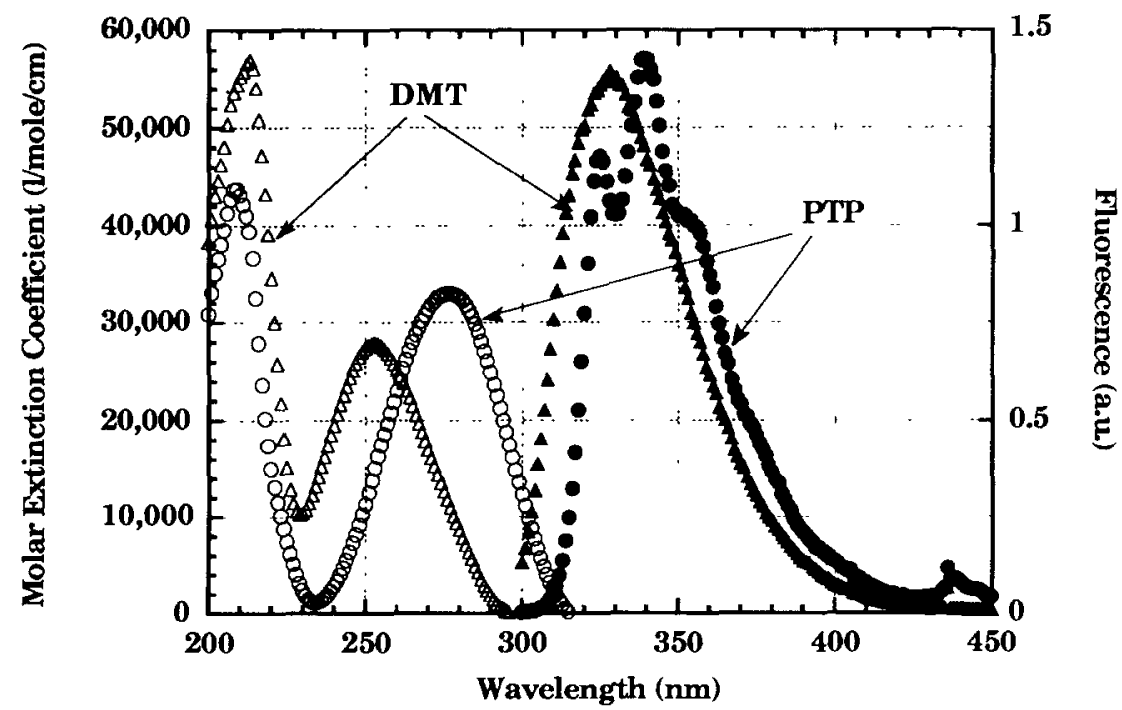

Fig. 4 Absorption and fluorescence spectra of PTP and DMT dissolved in cyclohexane Solıd symbols are tor the fluorescence emission data. Hollow symbols are the molar extmnction coefficient as described in the text 


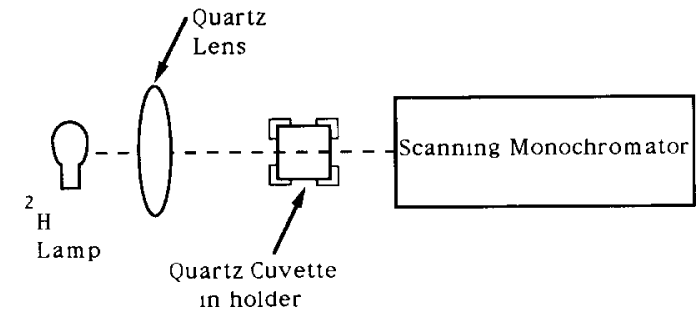

Fig. 5. Method used for measuring the molar extinction coefficient. Cuvette contained either solvent or solution of WLS and solvent.

\subsection{Fluorescence emission measurement}

The highest photon detection efficiency is achicved when the WLS emission peak wavelength corresponds with the maximum sensitivity of the PMT (typically $\approx 410 \mathrm{~nm}$ ). Spectral matching can be done either by a one shift system (unitary or binary system) or in a two shift system (ternary system). For these experiments we restricted our measurements to a binary system. Cyclohexane was chosen as the solvent because of its low absorptivity of incident energy.

A fluorometer was construced using the bandpass filtered deuterium lamp emissions as the excitation source. The bandpass filter has peak transmission at $222 \mathrm{~nm}$ and a FWHM of $25 \mathrm{~nm}$. The monochromator was equipped with a $500 \mathrm{~nm}$ blaze, 1200 grooves $/ \mathrm{mm}$ grating and the entrance and exit slits were set to have a resolution of $\approx 1 \mathrm{~nm}$. Similar to the absorption measurements, the WLS solution was contained in a fused silica cuvette. The luminescence of PTP and DMT at concentrations of $\approx 4.5 \times 10^{-5} \mathrm{M}$ in cyclohex- ane are shown as the solid symbols in fig. 4. The emission spectra have been corrected for the monochromator detection nonlinearities.

\subsection{Quantum efficiency measurement}

Luminescence quantum efficiency is defined as the probability that a molecule emits a photon after an absorption. Our measurements of the quantum efficiency were determined relative to a "known" standard using the relationship [15]:

$Q_{\mathrm{x}}=Q_{\mathrm{s}}\left(\frac{A\left(\lambda_{\mathrm{s}}\right)}{A\left(\lambda_{\mathrm{x}}\right)}\right)\left(\frac{I\left(\lambda_{\mathrm{s}}\right)}{I\left(\lambda_{\mathrm{x}}\right)}\right)\left(\frac{n_{\mathrm{x}}^{2}}{n_{\mathrm{s}}^{2}}\right)\left(\frac{D_{\mathrm{x}}}{D_{\mathrm{s}}}\right)$,

where $\mathrm{x}$ denotes the sample and $\mathrm{s}$ is the "known" standard solution, $A(\lambda)$ is the fraction of photons that were absorbed by the fluor at wavelength $\lambda, I(\lambda)$ is the relative intensity of excitation photons at $\lambda, n$ is the average index of refraction of the solution, and $D$ is the integrated area under the corrected emission spectrum. From eq. (2) the unknown quantum efficiency, $Q_{\mathrm{x}}$, is determined relative to a known quantum efficiency standard, $Q_{\mathrm{s}}$. Cyclohexane was used as the solvent and the solutions were sufficiently dilute, such that $n_{\mathrm{x}}^{2} / n_{\mathrm{s}}^{2} \approx 1$. The ratio $I\left(\lambda_{\mathrm{s}} / I\left(\lambda_{\mathrm{x}}\right)\right.$ is set equal to 1 because the emission spectra of the unknown and the standard are measured with the same excitation source (as described in section 3.2). The standard chosen was 9,10 diphenylanthracene (DPA) at a concentration of $3 \times 10^{-5} \mathrm{M}$. Relative to DPA $\left(Q_{\mathrm{f}}=0.86[16]\right)$ the luminescence quantum efficiency of DMT and PTP were determined to be 0.58 and 0.69 , respectively.

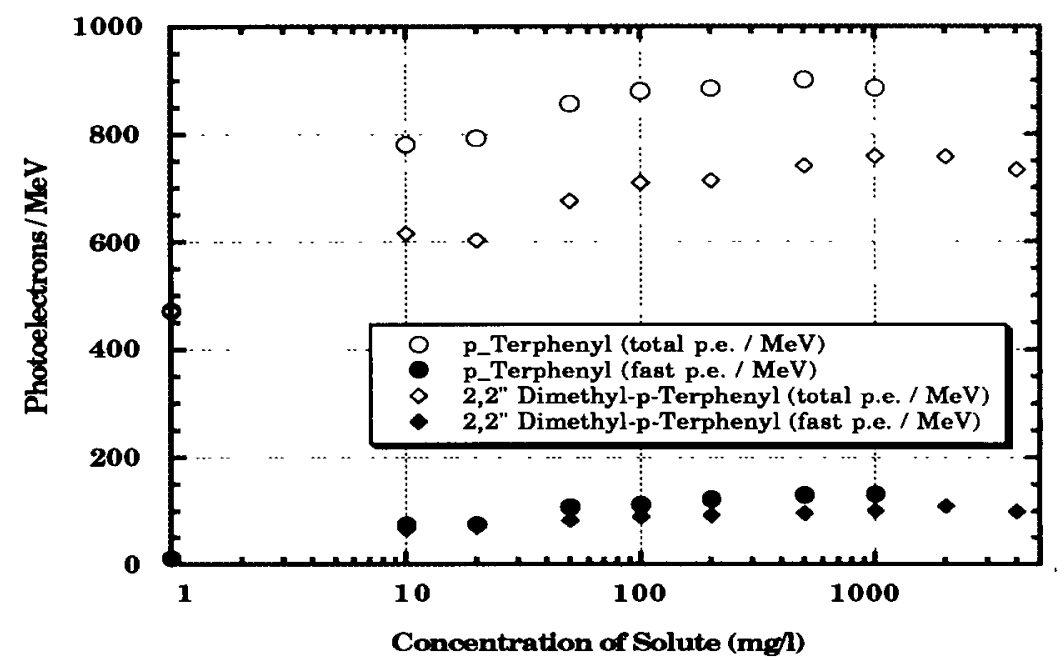

Fig. 6. Total measured photoelectron yield divided into the fast and slow components. $\mathrm{BaF}_{2}$ yields 227 fast photoelectrons $/ \mathrm{MeV}$ and 867 slow photoelectrons/ $\mathrm{MeV}$ when directly coupled to a fused silica end-window PMT. 


\subsection{Photoelectron yield measurements}

Photoelectron (PE) yield is a relative number that is typically reported to give an estimate of the size of the pulse to be measured. Inherent to determining the PE yield is the determination of the PMT gain. The procedure used to determine the PMT gain is outlined in O'Callaghan et al. [17] and Peak et al. [18]. The PMT gain is determined by first calibrating the multichannel analyzer in units of charge using the shaped square wave method [19]. Knowing the charge, $q$, corresponding to the mean value of the single photoelectron peak, the mean PMT gain can be determined using:

$$
\langle g\rangle=q /(N e)
$$

where $N(=1)$ is the number of photoelectrons generated in the photocathode, $e$ is the electron charge and $\langle g\rangle$ is the mean PMT gain. A Burle C31000M was used because the high gain GaP first dynode results in a well resolved single photoelectron peak ( $44 \%$ fullwidth-at-half-maximum). Biasing the C31000M PMT at $-1800 \mathrm{~V}$ gave a mean gain $(\langle g\rangle)$ of $8.0 \pm 0.2 \times 10^{6}$.

The PE yield of the $\mathrm{BaF}_{2} /$ WLS sample was determined using eq. (3) where now the only unknown in $N$. $\mathrm{BaF}_{2}$ directly coupled to the PMT yielded 1094 $\mathrm{PE} / \mathrm{MeV}$ for an amplifier shaping time of $3 \mu \mathrm{s}$. Table 1 summarizes the PE yield data. Measured PE yields
Table 1

Photoelectron yield and coincidence time spectra data

\begin{tabular}{|c|c|c|c|c|}
\hline Geometry & Slıde & $\begin{array}{l}\text { Total PE } \\
\text { yield } \\
{[\mathrm{PE} / \mathrm{MeV}]}\end{array}$ & $I_{\mathrm{f}} / I_{\mathrm{s}}$ & $\begin{array}{l}\text { FWHM } \\
\text { [ps] }\end{array}$ \\
\hline $\begin{array}{l}\text { Direct coupling } \\
\text { Cyclohexane }\end{array}$ & none & 1094 & 0.26 & 420 \\
\hline $\begin{array}{c}\text { in cuvette } \\
\text { PTP }(0.5 \mathrm{~g} / 1) \\
\text { cyclohexane }\end{array}$ & borosilicate & 370 & 0.022 & 1986 \\
\hline $\begin{array}{l}\text { DMT }(1.0 \mathrm{~g} / 1) \\
\text { cyclohexane } \\
\text { in cuvette }\end{array}$ & borosilicate & 762 & 0.154 & 796 \\
\hline
\end{tabular}

for a WLS solute dissolved at various concentrations in cyclohexane are shown in fig. 6. DETECT [20], an optical photon transport code, was used to determine the relative difference between the measured signal when the WLS has a thickness of $10 \mathrm{~mm}$ and an infinitesimal thickness. From the computer simulations, the PE yield was determined to increase by $30 \%$ when the cuvette filled with WLS was replaced with a translucent thin film of WLS having the same absorptivity. The WLS data presented in fig. 6 and table 1 have been renormalized to compensate for the losses

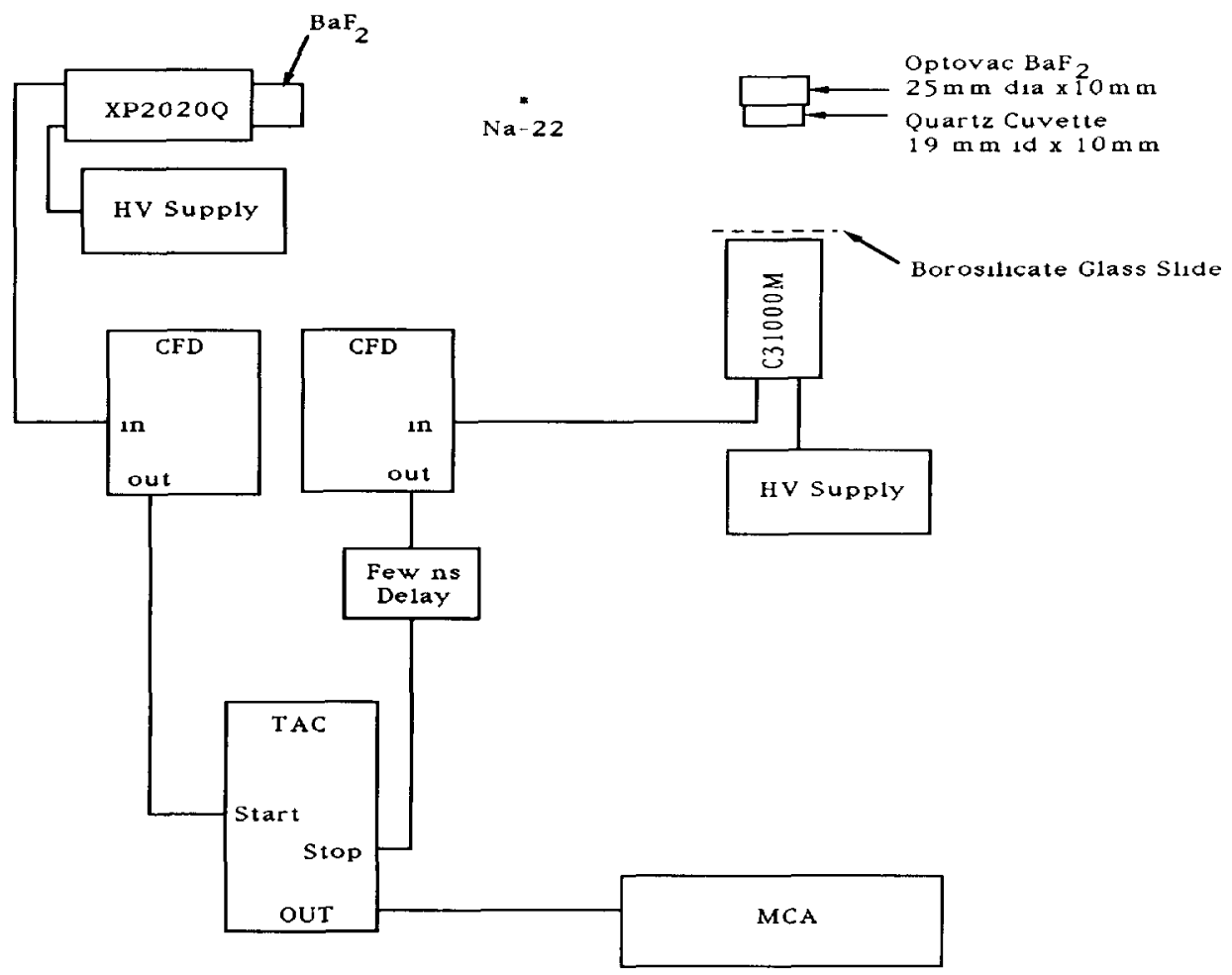

Fig. 7 Experimental schematıc used for measuring luminescence. 
imposed by the cuvette, actual measured values are $30 \%$ less than shown.

In fig. 6 , the increase in the total PE yield with increased solute concentration can be associated with increased wavelength shifting of the deep $\mathrm{UV} \mathrm{BaF}_{2}$ photons. At higher concentrations the total PE yield begins to decrease as a result of self-absorption by the solute and/or concentration quenching. The maximum measured concentration was $1.0 \mathrm{~g} / \mathrm{I}$ for PTP and 4.0 $\mathrm{g} / 1$ for DMT with the maximum PE yield at $0.5 \mathrm{~g} / 1$ for PTP and $1.0 \mathrm{~g} / 1$ for DMT.

\subsection{Luminescence time spectra measurements}

One of the most attractive characteristics of $\mathrm{BaF}_{2}$ is its fast, $0.8 \mathrm{~ns}$, scinitillation decay time constant. Ideally the WLS used should preserve this speed. The fluorescence decay time of the WLS was measured using the Bollinger and Thomas technique [21] with a modification by Moszyński and Bengston [22]. The pulse processing system used is shown in fig. 7. The $\mathrm{BaF}_{2}$ emissions serve as a pulsed UV source to the WLS. Fig. 8 shows the initial 100 ns of the luminescence pulse under various conditions. The peak at $\approx 18 \mathrm{~ns}$ primarily consists of the fast emission of $\mathrm{BaF}_{2}$ as well as the fast wavelength shifted emissions. The data beyond the peak value are fit to the sum of two exponentials corresponding to 1) the wavelength shifted fast emissions or the transmitted fast emissions, and 2) the sum of slow $\mathrm{BaF}_{2}$ and wavelength shifted slow $\mathrm{BaF}_{2}$ emissions. Note that the slow component from $\mathrm{BaF}_{2}$ is indistinguishable from the wavelength shifted slow component in these temporal measurements because the fluorescence decay time constant of the WLS is over two orders of magnitude faster than the slow
$\mathrm{BaF}_{2}$ decay time. The parent-prodigy relationship between the $\mathrm{BaF}_{2}$ slow emissions and the WLS emissions results in the apparent decay time constant that is the same as the parent.

To determine a decay time constant that is on the same order of magnitude as the temporal characteristics of the PMT, the response of the PMT must be unfolded from the measured luminescence time spectra. Similarly the PMT and fast $\mathrm{BaF}_{2}$ response must be unfolded from the measured WLS luminescence spectrum to determine the fluorescence decay time of the WLS. These deconvolutions were performed with a nonlinear least squares fitting routine [26], and the fluorescence decay time constants of DMT and PTP were determined to be $1.09 \pm 0.013 \mathrm{~ns}$ and $1.14 \pm 0.012$ ns, respectively.

The distributions shown in fig. 8 can also be used to determine the ratio of fast to slow intensities, $I_{\mathrm{f}} / I_{\mathrm{s}}$. The photon intensity, $I$, is obtained by integrating the functional form of the luminescence pulse using the parameters determined by the nonlinear least squares fit, including a correction for the part of the luminescence pulse that occurs earlier in time than the peak value. For $\mathrm{BaF}_{2}$ measured with the $\mathrm{C} 31000 \mathrm{M}$ PMT, the ratio of fast to slow photoelectrons was determined to be 0.26 , which is equivalent to 227 fast and 867 slow photoelectrons. This PE yield compares well with the results of Wei et al. [23]. When DMT and PTP luminescence spectra were measured with the borosilicate glass slide positioned between the sample and the PMT, $I_{\mathrm{f}}$ consists primarily of waveshifted fast $\mathrm{BaF}_{2}$ emissions, $I_{\mathrm{f}, \mathrm{wLS}}$. At low concentrations, $I_{\mathrm{s}}$ is the sum of transmitted slow $\mathrm{BaF}_{2}$ emissions, $I_{\mathrm{s}, \mathrm{BaF}_{2}}$, and wavelength shifted slow $\mathrm{BaF}_{2}$ emissions, $I_{\mathrm{s}, \mathrm{WLs}}$. At higher concentrations, the deep UV emissions of $\mathrm{BaF}_{2}$ are

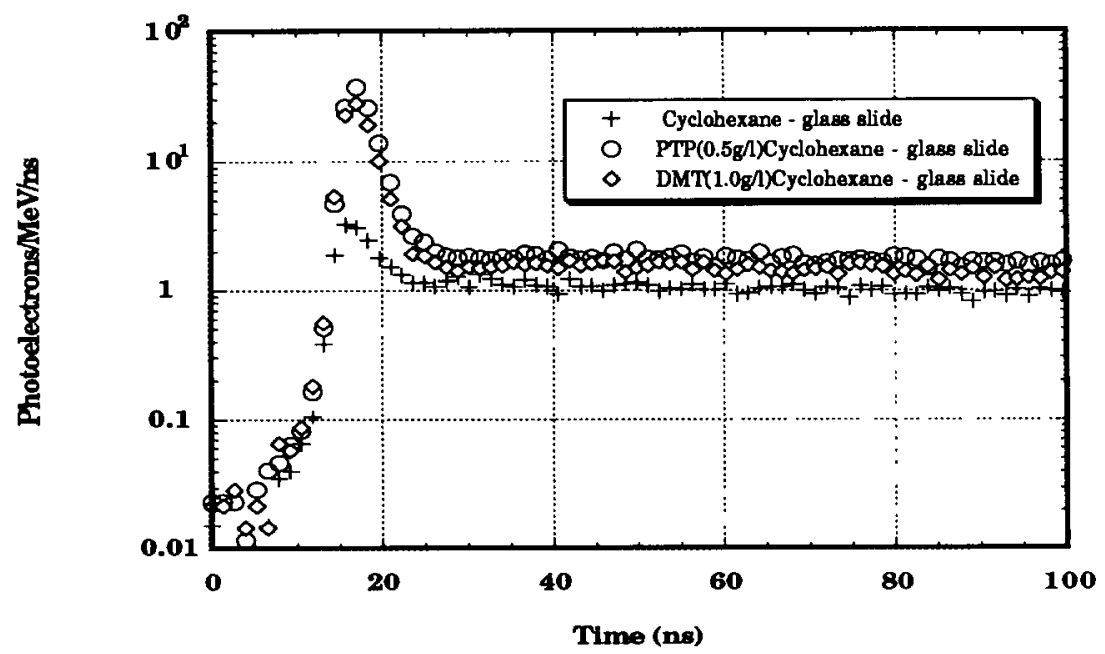

Fig. 8. Luminescence time spectra for PTP and DMT dissolved in cyclohexane. Data was acquired in 0.262 ns bins, but rebinned to $1.310 \mathrm{~ns}$ bins for display. 


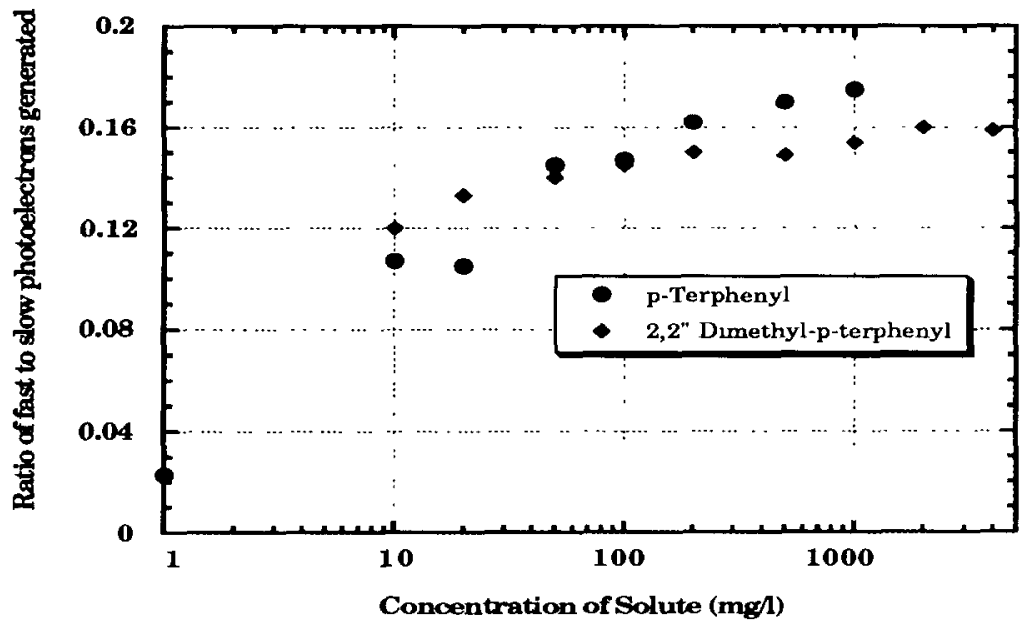

Fig. 9. Ratio of fast to slow photoelectrons generated in the PMT as a function of solute concentration in cyclohexane

fully absorbed by the WLS. Thus, as the concentration is increased, $I_{\mathrm{f}} / I_{\mathrm{s}}$ initially increases and then saturates at a value that is WLS dependent (see fig. 9).

\subsection{Coincidence time spectra measurements}

The time resolution or coincidence time width is a figure of merit used in time spectroscopy. The coincidence time width is inversely proportional to the intensity of detected photons at the beginning of the pulse, $I_{0}(t)$, as well as system noise. $I_{0}(t)$ is proportional to the total number of photoelectrons detected and inversely proportional to the decay time constant; therefore, the coincidence time width increases with increased decay time constant and decreased number of photoelectrons [24].

The pulse processing electronics used to make time resolution measurements are similar to those shown in fig. 7 except the stop signal is generated by the $\mathrm{BaF}_{2}$ directly coupled to either the PMT or with an intermediate glass slide and/or cuvette. For these measurements the start detector was a Phillips XP2020Q PMT coupled to a $25 \mathrm{~mm}$ diameter $\times 10 \mathrm{~mm}$ right circular cylinder of $\mathrm{BaF}_{2} . \mathrm{BaF}_{2}$ coupled directly to the $\mathrm{C} 31000 \mathrm{M}$ (stop) PMT resulted in the narrowest time coincidence width, 420 ps FWHM. Introducing a borosilicate slide and a cuvette filled with cyclohexane between the $\mathrm{BaF}_{2}$ crystal and the C31000M PMT increased the time coincidence width to 1986 ps FWHM. Introducing DMT at $1.0 \mathrm{~g} / 1$ or PTP at $0.5 \mathrm{~g} / 1$ into the cuvette decreased this width to $796 \mathrm{~ns}$ and $691 \mathrm{~ns}$, respectively. Table 1 presents a summary of the results.

\section{Spectral separation using wavelength shifters}

In the previous section, measurements of the most important properties necessary for wavelength shifting
$\mathrm{BaF}_{2}$ emissions were presented. These measurements showed PTP and DMT are fairly good choices for shifting the fast emission of $\mathrm{BaF}_{2}$ above $300 \mathrm{~nm}$. However, for high count rate applications, it may be advantageous to collect only the fast photons and eliminate the slow. To spectrally separate $\mathrm{BaF}_{2}$ emissions with PTP and DMT, the detection geometry illustrated in fig. 10 was used. The WLS adsorbs primarily the fast emissions while transmitting the slow emissions. The WLS luminesces isotropically, so a fraction of the photons can be detected perpendicular to the axis of the scintillator. For maximum spectral separation a reflector around the WLS cannot be used, reducing the light collection efficiency. Using the perpendicular configuration the ratio of fast to slow photoelectrons is 0.27 and 0.33 for PTP and DMT respectively, at their optimal concentrations. For DMT, the fast photoelectrons yield is $\approx 50$ photoelectrons $/ \mathrm{MeV}$ [25]. Since PTP has a higher QE one would expect the fast photoclectrons yield to be higher, but in either case the in-line measurement resulted in the detection of twice as many fast photoelectrons. Because of the photophysical properties of WLS fluors, better spectral separa-

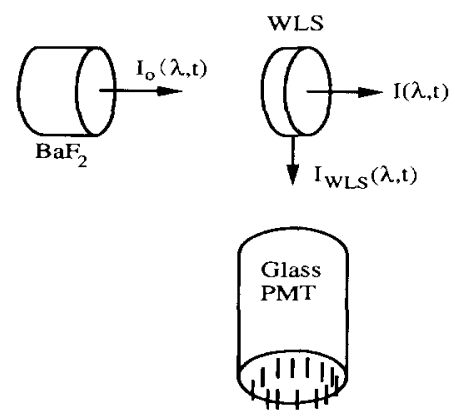

Fig. 10. Detection geometry used to fachlitate spectral separation of $\mathrm{BaF}_{2}$ emissions using PTP or DMT. 
Table 2

Summary of measurements

\begin{tabular}{|c|c|c|c|}
\hline Properties & PTP $^{b}$ & $\mathrm{DMT}^{\mathrm{b}}$ & $\mathrm{BaF}_{2}{ }^{\mathrm{c}}$ \\
\hline Peak absorption wavelength [nm] & 275 & 252 & \\
\hline$\epsilon\left(\lambda_{\text {peak }}\right)$ & 32000 & 29000 & \\
\hline Peak fluorescence wavelength [nm] & 340 & 325 & \\
\hline Quantum efficiency, $Q_{\mathrm{f}}$ & 0.69 & 0.58 & \\
\hline Optımal concentration $[\mathrm{g} / \mathrm{l}]$ & 0.5 & 1.0 & \\
\hline $\mathrm{PE} / \mathrm{MeV}$ & 902 a & $762 \triangle$ & 1094 \\
\hline Fluorescence decay time constant [ns] & $1.136^{\mathrm{a}}$ & $1.088^{a}$ & 0.8 \\
\hline Ratio of fast to slow PE, in-line measurement ${ }^{d}$ & $0.17^{\mathrm{d}}$ & $0.15^{\mathrm{a}}$ & 026 \\
\hline Ratio of fast to slow PE, in-line measurement ${ }^{e}$ & & & 0.12 \\
\hline Ratio of fast to slow PE, perpendicular measurement ${ }^{d}$ & $0.27^{\mathrm{d}}$ & $0.33^{a}$ & \\
\hline Coincidence time width $[\mathrm{ps}]$ & 691 a & $796 d$ & 420 \\
\hline
\end{tabular}

a Determined at optimal concentration.

b Measured with a "borosilicate" PMT.

" Measured with a fused silica PMT.

'Determined from timing measurement.

' Determined from emission spectrum (fig. 2) fast: $200 \leq \lambda \leq 240$, slow: $241 \leq \lambda \leq 400$.

tion can be realized only at the expense of decreased $\mathrm{QE}$ and increased decay time [9].

\section{Conclusion}

Table 2 summarizes the measured values of the quantities that are necessary to characterize an organic fluor to be used as a WLS for $\mathrm{BaF}_{2}$. The fluorescence quantum efficiency and the fluorescence decay time constant are the most critical. It has been shown that PTP is a better candidate as a WLS for $\mathrm{BaF}_{2}$ than DMT, but DMT facilitates better spectral separation. If a high fluorescence quantum efficiency WLS is used, the total number of photoelectrons detected per luminescence pulse may approach that of a $\mathrm{BaF}_{2}$ crystal directly coupled to a fused silica end-window PMT. The sacrifice that must be made is a marginally slower signal. WLSs may also be used to spectrally separate the $\mathrm{BaF}_{2}$ emissions, but only marginal separation can be achieved using the perpendicular detection geometry. In general, spectral separation using this geometry is limited due to photophysical properties of WLS molecules.

\section{Acknowledgements}

The authors wish to thank Dr. Raoul Kopelman for his assistance on organic molecular photophysics, Dr. Mary Brake for use of her monochromator, Dr. William W. Moses for his help in establishing our confidence in the determination of the time spectra, Dr. Stephen Derenzo for use of his nonlinear least squares fitting program, and John Valentine and Valentin Jordanov for their assistance in calibrating our MCA to units of charge. T.A. DeVol performed this research while under appointment to the Environmental Restoration and Waste Management Fellowship Program, which is administered by Oak Ridge Associated Universities for the U.S. Department of Energy.

\section{References}

[1] A. Tzalmona and P.S. Pershan, Phys. Rev. 182 (1969) 906.

[2] S. Majewski and M.K. Bentley, Nucl. Instr. and Meth. A260 (1987) 373.

[3] M. Laval et al., Nucl. Instr. and Meth. 206 (1983) 169.

[4] H. Kobayashi et al., Nucl. Instr. and Meth. A270 (1988) 106

[5] E. Dafni, Nucl. Instr. and Meth. A254 (1987) 54.

[6] W. Klamra, Th. Lindblad, M. Moszyński, L.O. Norlin and J. Weimer, Nucl. Instr. and Meth. A265 (1988) 485.

[7] V. Yanovsky and P. Kozma, Nucl. Instr. and Meth. A276 (1989) 659.

[8] E. Lorenz, G. Mageras and H. Vogel, Nucl. Instr. and Meth. A249 (1986) 235.

[9] H.O. Wirth, F.U. Herrman, G. Herrmann and W. Kern, Molecular Crystals 4 (1968) 321.

[10] M. Bowen, S. Majewski, D. Pettey, J. Walker, R. Wojcik and C. Zorn, IEEE Trans. Nucl. Sci. NS-36 (1989) 562

[11] P. Schotanus, P. Dorenbos, C.W.E. Van Eijk and H.J. Lamfers, Nucl. Instr. and Meth A281 (1989) 162.

[12] P. Schotanus, C.W.E. Van Eijk, R.W. Hollander and J. Pijpelink, Nucl. Instr and Meth. A238 (1985) 564

[13] I.B. Berlman, Handbook of Fluorescence Spectra of Aromatic Molecules (Academic Press, 1971).

[14] C.A. Parker, Photoluminescence of Solutions (Elsevier, 1968). 
[15] J.N. Demas and G A Crosby, J. Phys. Chem 75 (1971) 991

[16] J V. Morris, M.A. Mahaney and J.R. Huber, J. Phys. Chem. 80 (1976) 969.

[17] J.P O'Callaghan, R. Stanek and L.G. Hyman, Nucl. Instr. and Meth. 225 (1984) 153.

[18] L.S. Peak and P. Soler, Nucl. Instr. and Meth. A301 (1991) 341.

[19] J. Valentıne, V. Jordanov, D. Wehe and G. Knoll, Nucl Instr. and Meth. A314 (1992) 119.

[20] G.F. Knoll, T.F. Knoll and T.M. Henderson, IEEE Trans Nuc. Sc1. NS-35 (1988) 872.
[21] L.M. Bollinger and G.E. Thomas, Rev. Sc1. Instr. 32 (1961) 1044

[22] M. Moszyńskı, and B. Bengtson, Nucl. Instr, and Meth. 142 (1977) 417.

[23] Z.Y. Wei, R Y Zhu, H. Newman and Z.W. Yin, Nucl Instr. and Meth. B61 (1991) 61.

[24] B. Sigfridsson, Nucl. Instr. and Meth. 54 (1967) 13.

[25] E. Lorenz, Max Planck Institut für Physık, private communication.

[26] S.E. Derengo, Lawrence Berkeley Laboratory Report, LBL-33050 (1993). 